\title{
A randomised controlled trial in diabetes demonstrating the positive impact of a patient activation strategy on diabetes processes and HbA1c: the WICKED project
}

\author{
SYED MR GILLANI, ${ }^{1}$ ALAN NEVILL, ${ }^{2}$ BALDEV M SINGH ${ }^{1}$
}

\begin{abstract}
Background: Patient activation is a demonstration of people participating effectively in their own care as measurable in objective outcomes. Techniques of activating patients are various.

Aims: We developed a structured information booklet to promote patient activation and report the 1-year outcomes of a randomised controlled trial assessing its impact on diabetes care processes and on glycaemic control.

Design and setting: It is an open label cluster randomised trial involving all people with diabetes aged more than 18 years within Wolverhampton Clinical Commissioning Group.

Methods: All people with diabetes were cluster randomised into a group who were multiply mailed (MM) at 0,3 and 6 months whilst a control group was mailed once at 3 months. Comparison of a Failed Process Score (FPS) between active and control groups was performed at 0, 3 and 12 months and of $\mathrm{HbA}_{1 \mathrm{c}}$ at baseline and 12 months.

Results: FPS improved significantly with multiple mailing $(p=0.013)$, with particular impact on those with poor baseline FPS ( $\geq 2$ ) (achieved FPS $\leq 1$ at 12 months $49.2 \%$ vs. $46.0 \%$, $X^{2}=6.09, p<0.05$ ). Overall $\mathrm{HbA}_{1 c} \%$ across the year (adjusted) was significantly better with $\mathrm{MM}(\mathrm{p}=0.021)$, with specific impact in those with a baseline $\mathrm{HbA}_{1 \mathrm{c}} \leq 7.5$ (MM HbA $\mathrm{H}_{1 \mathrm{c}} \%$ $6.7 \pm 0.07$ (mean \pm SEM) vs. $7.0 \pm 0.09$; mean \pm SEM difference $0.3 \pm 0.1, F=11.1, p=0.009$ ).

Conclusion: The direct provision of structured information to people with diabetes activates them to engage in their care delivery as reflected in care process and glycaemic control outcomes.
\end{abstract}

Br J Diabetes 2017;17:58-63

\footnotetext{
New Cross Hospital, Wolverhampton, UK

2 University of Wolverhampton, Wolverhampton, UK

Address for correspondence: Dr Syed MR Gillani

Wolverhampton Diabetes Centre, New Cross Hospital, Wednesfield Road,

Wolverhampton WV10 0QP, UK

Tel: +44 (0)1902 695313

E-mail: Syed.gillani@nhs.net

http://dx.doi.org/10.15277/bjd.2017.134
}

Key words: diabetes, care delivery, patient activation, patient engagement, key care processes

\section{Introduction}

Diabetes is a costly worldwide epidemic that requires further exploration of the concepts of patient engagement, enablement and empowerment and partnership working to activate people to self-care, ${ }^{1}$ and thus contribute to the amelioration of the many personal and societal aspects of the burden of the disease. ${ }^{2,3}$ Patient activation is a broad behavioural concept, ${ }^{4}$ and its intent is to encourage people with long-term conditions such as diabetes to acquire the knowledge, skills and confidence to participate actively but also effectively in their own disease management as measurable in defined outcomes. A number of user-focused non-pharmacological interventions have demonstrated an effect on diabetes-specific surrogate markers, ${ }^{5}$ and there is evidence that increased patient activation is associated with better behaviours and outcomes. ${ }^{6}$ As such, it perhaps should be considered as an outcome of diabetes care provision in its own right. ${ }^{7}$

One traditional mechanism is to promote knowledge acquisition through structured education programmes, but they are costly, with uncertain outcomes and they have very poor uptake in the UK.8,9 Another technique is the provision of structured and easy to understand information directly to users in an attempt to instigate action though self-directed reflective learning, ${ }^{10}$ which more recently has been encapsulated in the 'Information prescription' initiative. ${ }^{11}$ It is not our intention in this paper to suggest that one mechanism is better than the other, nor have we compared these two methodologies, and both aspire to modify behaviour. Our focus is on the use of information.

In our local model of diabetes care, WICKED (Wolverhampton Interface Care, Knowledge Empowered Diabetes), we have determined that it should be user-centric, that patient activation is a service objective, that patients have a right to their own specific information, that patients should have the opportunity to use that information to improve their care and to liaise in equal and informed partnership with those providers of care by establishing their agreed care plans in a recognised process of care planning..$^{12}$ We also understand, as will any larger-scale provider, that in our local health economy of around 265,000 people, with more than 17,000 people with known diabetes and a high incidence rate of new diabetes, we must deliver any mechanism systematically and 
equitably, without exclusion or exception, and cheaply so as not to embarrass resource requirements, and effectively so that it must be evidenced to be of benefit. Yet there is no known trial to evaluate the impact of provision of individualised diabetes-specific information to people with diabetes, agnostic of their attitudes, aptitudes or degree of engagement with the health service, on any measure of patient engagement.

Having published preliminary outcomes, ${ }^{13}$ we are now presenting the end study full year findings of this large randomised controlled trial to determine the impact of the universal provision of diabetesspecific information on patient activation as measured by the rate of completion of key care processes in diabetes and, in addition, on the key diabetes surrogate marker of glycaemic control, $\mathrm{HbA}_{1 \mathrm{c}}$.

\section{Methods}

As previously described, ${ }^{13}$ a systematically designed, structured and individualised report containing their core key diabetesrelated information, which was the intervention called 'My Diabetes, My Information, My Plan' (available at www.wdconline.org.uk, attached as appendix 1), was mail delivered across our entire health economy to people with diabetes divided into two groups according to a cluster randomisation protocol. ${ }^{13}$

The recording or measurement of nine key diabetes process measures and their outcomes were analysed $\left(\mathrm{HbA}_{1 c}\right.$, systolic blood pressure, cholesterol or cholesterol/HDL cholesterol ratio, body mass index (BMI), recording of smoking status, retinal screening, urinary albumin creatinine ratio, serum creatinine, foot examination). For process outcomes, a reading within 15 months was taken as positive. The Failed Process Score (FPS) was 0 if all measures were attained within 15 months, and 9 meant all failed to be measured.

The positive 3-month impact of a single mailing versus no mailing on diabetes process measure outcomes, measured as the FPS, which was taken as a marker of patient activation, has already been published. ${ }^{13}$

At the end of 3 months, all people in the control group were crossed over to receive the information booklet for the first time and for once only (single mailed, SM). People in the active group received the booklet a second time at 3 months and then finally a third time at 6 months (multiply mailed, MM).

Both groups were followed up for 12 months from baseline. We continued to accrue data on a rolling monthly basis, ensuring systematic quality in data capture as is our routine service practice, ${ }^{14}$ but it was not analysed until the end of the 12-month period. Therefore, the final analysis is a comparison of multiple mailings $(n=3)$ delivered at baseline, 3 and 6 months versus a single mailing undertaken at 3 months. Compared with the baseline population, at the end of 12 months 866 people in total were lost to ascertainment (453 deceased, 378 moved away and 35 not traceable), leaving a final cohort of 13,956 people at the end of 12 months of the trial period.

A complete log of all failed deliveries returned back to the department and all enquiry phone calls received was kept, but this was not subject to any form of analysis as it was less than $1 \%$ and unlikely to be of any meaningful significance.
Results are presented as the mean \pm standard deviation (SD) unless otherwise stated.

All data were analysed on SPSS version 22 , with the results of statistical tests taken as significant at $p<0.05$. Comparison of means was by the Student's $t$ test or by Mann-Whitney $U$ tests for parametric and non-parametric data, respectively. Differences between proportions were analysed using the $\mathrm{X}^{2}$ test and analysis of the effects of confounding factors was by univariate or binary logistic regression analysis for ordinal or categorical data, respectively.

The study was registered in the UK national research database (UK CRN ref: DRN 795, available at http://public. ukcrn.org.uk/Search/StudyDetail.aspx?StudyID=14324) and US clinical trials database (Clinical Trials Registration: NCT02200965). Ethical permission was obtained from NHS Health Research Authority (NRES committee North East-York, REC Ref: 13/NE/0052) and further clarification was obtained from the National Information Governance Board.

\section{Results}

\section{Demographics}

Demographic and clinical data are presented in Table 1, noting the minor differences between groups for deprivation score, BMI, systolic blood pressure and coronary heart disease risk status.

Table 1 Demographics and clinical parameters of those receiving multiple mailings of a structured information booklet (MM, 0, 3 and 6 months) compared with those mailed singly (SM, 3 months)

\begin{tabular}{llll}
\hline Demographic data & $\begin{array}{l}\text { MM } \\
\mathbf{( N = 8 , 0 4 5 )}\end{array}$ & $\begin{array}{l}\text { SM } \\
(\mathbf{N = 5 , 9 1 1 )}\end{array}$ & P value \\
Age (years) & $63.9 \pm 14.5$ & $63.4 \pm 14.4$ & NS \\
Sex (male) & $54 \%$ & $55 \%$ & NS \\
Ethnicity (white) & $69 \%$ & $70 \%$ & NS \\
Deprivation score & $35.2 \pm 15.7$ & $35.9 \pm 16.6$ & $<0.05$ \\
Type 2 diabetes & $94 \%$ & $94 \%$ & NS \\
Duration of diabetes & $10.4 \pm 8.4$ & $10.5 \pm 8.7$ & NS \\
Smoking (never smoked) & $60 \%$ & $60 \%$ & NS \\
& & & \\
Clinical parameters & & & \\
Body mass index (kg/m²) & $30.9 \pm 6.3$ & $31.1 \pm 6.3$ & $<0.05$ \\
BP (mm Hg) & $135 \pm 16$ & $132 \pm 16$ & $<0.001$ \\
HbA 1 DCCT (\% glycated) & $7.8 \pm 1.7$ & $7.8 \pm 1.7$ & NS \\
HbA 1 IFC (mmol/mol) & $61.5 \pm 18.1$ & $61.6 \pm 18.3$ & NS \\
Urine ACR (mg/mmol) & $8.7 \pm 34.6$ & $8.9 \pm 43.9$ & NS \\
Creatinine ( $\mu$ mol/l) & $88.3 \pm 43.2$ & $89.1 \pm 46.4$ & NS \\
Cholesterol/HDL cholesterol ratio & $3.8 \pm 1.4$ & $3.8 \pm 1.4$ & NS \\
Cardiovascular status (primary risk) & $71 \%$ & $69 \%$ & $<0.01$ \\
10 year Framingham CHD risk (\%) & $18.0 \pm 7.5$ & $17.8 \pm 7.4$ & NS \\
Any retinopathy & $58 \%$ & $57 \%$ & NS \\
Any foot risk (intermediate or high) & $57 \%$ & $57 \%$ & NS \\
\hline
\end{tabular}

Results are mean \pm SD or percentages.

$A C R$, albumin creatinine ratio; $C H D$, coronary heart disease; $D C C T$, Diabetes Control and Complication Trial; IFC, International Federation of Clinical Chemistry. 
Table 2 Comparison of the Failed Process Score (FPS) between those multiply mailed (MM, 3 mailings) and those singly mailed (SM) in the whole cohort or those with a baseline FPS $\leq 1$ or $\geq 2$

\begin{tabular}{lllll}
\hline & & MM & SM & P value \\
FPS whole cohort & $\mathrm{N}=8,045$ & $\mathrm{~N}=5,911$ & \\
& Baseline & $1.70 \pm 1.78$ & $1.71 \pm 1.81$ & NS \\
& 12 months & $1.65 \pm 1.92$ & $1.72 \pm 1.95$ & 0.013 \\
FPS $\leq 1$ & N=4,665 & $\mathrm{N}=3,412$ & & \\
& Baseline & $0.50 \pm 0.50$ & $0.40 \pm 0.50$ & NS \\
& 12 months & $1.23 \pm 1.47$ & $1.24 \pm 1.50$ & NS \\
FPS $\geq 2$ & N=3,380 & N=2,499 & & \\
& Baseline & $3.35 \pm 1.58$ & $3.38 \pm 1.61$ & NS \\
& 12 months & $2.25 \pm 2.30$ & $2.38 \pm 2.28$ & 0.002
\end{tabular}

Results are means \pm SD and are analysed by the Mann-Whitney $\mathrm{U}$ test.

\section{The Failed Process Score (FPS)}

The mean baseline FPS was not significantly different between groups (Table 2), whilst mean FPS was significantly lower at 12 months in those multiply mailed $(p=0.013)$. For those with a baseline FPS $\leq 1$, neither the baseline FPS score nor the 12-month FPS score was different between groups, nor was there any significant difference in the proportion that deteriorated to FPS $\geq 2$ (MM 28\% vs. SM 28\%, NS). However, for those with a baseline FPS $\geq 2$, the mean baseline FPS was similar but, at 12 months, FPS was significantly better in those multiply mailed $(p=0.002)$ and significantly more attained a good FPS category of $\leq 1$ (MM $49 \%$ vs. SM $46 \%$; $\left.X^{2}=6.09, p=0.014\right)$. In those with baseline FPS $\geq 2$, in binary logistic regression $\left(X^{2}=370.9, p<0.001\right)$, significant fac- tors for attaining 12 months FPS $\leq 1$ were baseline FPS $(p<0.001)$, gender $(p=0.022)$ and MM $(p=0.028)$, such that the likelihood ratio of achieving the good attainment category of FPS $\leq 1$ with MM was 1.15 (95\% Cl 1.02 to 1.29) compared with SM.

$\mathrm{HbA}_{1 \mathrm{c}}$

We selected those who had both a baseline $\mathrm{HbA}_{1 \mathrm{c}}$ measure and a repeat $\mathrm{HbA}_{1 \mathrm{c}}$ at least 6 months after the initial mailing date ( $n=10,015, M M 5,637, S M 4,378)$. Their baseline characteristics showed no significant difference for age, gender, ethnicity, type of diabetes, duration of diabetes, baseline FPS score or baseline $\%$ glycated $\mathrm{HbA}_{1 \mathrm{c}}$ (MM $7.8 \pm 1.64$ vs. $7.8 \pm 1.62$ ), but small but significant differences were found for the index of deprivation (MM 34.7 \pm 15.8 vs. $35.9 \pm 16.5, \mathrm{p}=0.001$ ) and BMI (MM 30.8 \pm 6.1 vs. SM $\left.31.2 \pm 6.2 \mathrm{~kg} / \mathrm{m}^{2}, \mathrm{p}=0.013\right)$. In the whole cohort, $\mathrm{HbA}_{1 \mathrm{c}}$ improved over the year (baseline $\mathrm{HbA}_{1 c} \% 7.8 \pm 1.6$ vs. final $\mathrm{HbA}_{1 c} \% 7.5 \pm 1.6, \mathrm{p}<0.001$ ) (Figure 1, left panel). The crude end year $\mathrm{HbA}_{1 \mathrm{c}}$ was not significantly different between groups (MM $7.5 \pm 1.6$ vs. SM 7.6 \pm 1.6, NS). However, adjusting for relevant factors (univariate regression analysis, $F=54.4, r^{2}=0.12, p<0.001$ : age $(p<0.001)$; duration of diabetes $(p<0.001)$, BMI $(p<0.001)$ but gender, ethnicity and type of diabetes all non-significant), then the MM group differed significantly from the SM group $(F=5.32, p=0.021)$ with an adjusted mean \pm SEM difference of $-0.2 \pm 0.08 \mathrm{HbA}_{1} \mathrm{c} \%$. Introducing baseline $\mathrm{HbA}_{1 c} \%$ categories as $\leq 7.5,7.6-8.4$ and $\geq 8.5$ into the model $\left(F=99.9, r^{2}=0.40\right.$, $p<0.001$ : age, $p<0.001$; duration of diabetes, $p<0.001$; gender, ethnicity, IMD score, BMI and type of diabetes all not significant) showed the impact of being multiply mailed remained significant ( $F=3.97, p=0.046)$, but with a strong effect according to baseline $\mathrm{HbA}_{1 c}$ category ( $\left.\mathrm{F}=363.9, \mathrm{p}<0.001\right)$. The significant point difference lay amongst those in the baseline $\mathrm{HbA}_{1 c}$ category $\leq 7.5$ (MM

Figure 1. Mean (left panel) and delta (right panel) $\mathrm{HbA}_{1 \mathrm{c}}$ outcomes at the end of 12 months categorised by baseline $\mathrm{Hb} \mathrm{A}_{1 \mathrm{c}}$ status in those receiving multiple versus single mailings
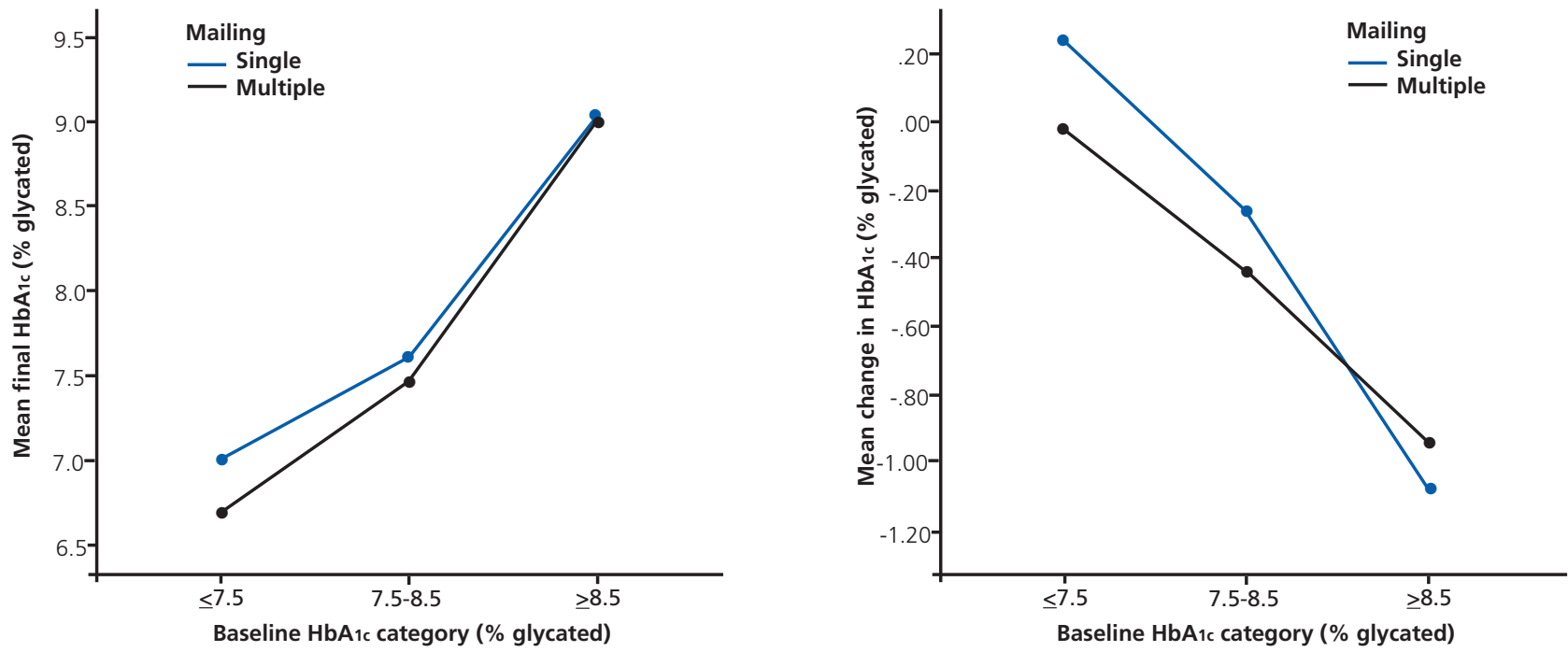
$\mathrm{HbA} 1 c \% 6.7 \pm 0.07$ vs. $7.0 \pm 0.09$, mean \pm SEM difference $0.3 \pm 0.1$, $\mathrm{F}=11.1, \mathrm{p}=0.009$ ). Analysis of the change between final and initial $\mathrm{HbA}_{1 \mathrm{c}}$ values by $\mathrm{HbA}_{1 \mathrm{c}}$ category (Figure 1, right panel) showed this to be an improvement or at least avoidance of deterioration of $\mathrm{HbA}_{1 \mathrm{c}}$ levels in the MM group in the $\mathrm{HbA}_{1 \mathrm{c}} \%$ category $\leq 7.5$, amongst whom the delta $\mathrm{HbA}_{1 c} \%$ was $-0.07 \pm 0.07$ vs. $0.20 \pm 0.08$ (delta $\mathrm{HbA}_{1 c} 0.3 \pm 0.1, \mathrm{~F}=7.05$, $\mathrm{p}=0.008$ ).

\section{Discussion}

The positive outcomes for the impact of a patient activation tool, 'My Diabetes, My Information, My Plan', on diabetes process attainment (FPS) and glycaemic control $\left(\mathrm{HbA}_{1 \mathrm{c}}\right)$ are novel. They should be considered in relation to the potential to benefit as well as the balance of the likely impact of patient activation versus the magnitude of service inactivation.

For the FPS, in the overall cohort the cyclical impact of the UK primary care Quality and Outcomes Framework (QOF), in which maximal service effort is exerted to complete the key diabetes process measures in order to achieve end of year financial reimbursement, can easily be discerned. Both groups showed a significant improvement over the QOF time frame, but with a significant better attainment in the MM group, meaning that a discernible effect was demonstrated in the face of performance-managed service activation. In the whole cohort, almost $60 \%$ were already in a high attainment position and thus could not be further benefited. When considering those with poorer baseline attainment (FPS $\geq 2$ ), at the end of 12 months they were $15 \%$ relatively more likely to be in the higher attainment category (FPS $\geq 1$ ). Thus, the impact of SM (as initially published) and then MM versus SM can be seen to have achieved its objective of having a significant impact over and above that of the current maximal driver of diabetes service activation namely QOF - and to have separately benefited the intended target groups in lower attainment categories.

The impact on $\mathrm{HbA}_{1 \mathrm{c}}$ can be similarly considered. Our a priori expectation was that, if any differential impact were to have occurred, it would have been in those with poorer baseline glycaemic control but, in hindsight, the opposite outcome is perhaps both predictable and understandable. As is well recognised, the focus of clinicians and services will be on poorly controlled patients; 5,15 service inertia and delay is a crucial reason for poor attainment in such patients 16,17 and the propensity to improve is almost certainly dependent on service intervention through drug titration and, in many patients, escalation to injectable therapies..$^{18} \mathrm{~A}$ patient in this category is unlikely to have been able to influence their own outcome over and above the impact of service interventions. However, in the better baseline $\mathrm{HbA}_{1 \mathrm{c}}$ attainment cohort $\left(\mathrm{HbA}_{1 \mathrm{c}} \leq 7.5 \%\right)$, the service would have been less focused on them as they were already at or below the UK HbA 1 c attainment target, ${ }^{19}$ there would have been no perception that drug therapies required modification, and patients would have been more likely to have been able to significantly modify their own already good attainment perhaps by diet and life style interventions or by improved concordance. The MM group essentially maintained their $\mathrm{HbA}_{1 c}$ whilst the comparators deteriorated relatively by $0.3 \mathrm{HbA}_{1 c} \%$. This seems small, but roughly equates to half of the size of effect of the addition of a second- or third-line oral hypoglycaemic agent in type 2 diabetes ${ }^{20}$ but, in this case, a benefit deliverable to thousands of patients. It is the same - if not better - magnitude of effect as is seen in highly structured education programmes such as DESMOND or DAFNE. ${ }^{21,22}$ It is regrettable that we are not able to comment on the potential impact of SM, or otherwise MM, compared with not being mailed at all, but a logical assumption is that MM would have achieved some degree of greater benefit if it had been compared with no mailing at all. We have already addressed the question as to where a SM has benefit (to FPS) and now show that MM has added benefit over $\mathrm{SM}$ (to FPS and $\mathrm{HbA}_{1 \mathrm{c}}$ ).

\section{Comparison with existing literature}

The small size of any magnitude of effect can be further considered in the light of known evidence. In a recent meta-analysis, non-pharmacological interventions were extensively reviewed ${ }^{5}$ and they can be categorised into three categories of quality improvement strategies targeting health systems, healthcare providers and people with diabetes. It was concluded in this review that health system-wide interventions and patient-focused strategies are more likely to influence outcomes in low to intermediate risk groups, while the high-risk group gets most benefit from strategies focusing on interventions by healthcare professionals, pointing to the possible beneficial impact and increasing need for service activation in this high-risk group. ${ }^{16,17}$ In this context, all such non-pharmacological interventions have only shown modest improvements in hard (albeit surrogate) outcomes such as $\mathrm{HbA}_{1 c}$ of the average magnitude of $0.37 \%$, precisely in line with the magnitude of benefit that we currently demonstrate.

\section{Strengths and limitations}

The strength of the study is its large size, no selection bias and a cluster randomised design that made it possible to evidence this intervention in a highly complex care delivery structure of the NHS.

The limitations of the study are acknowledged to be the relatively small magnitude of change observed, the relatively short time frame to first assessment of the MM versus SM groups and the inability to assess hard longer term clinical outcomes within that time frame. It is possible that the reported observed benefit at 3 months could have been by chance or random finding, ${ }^{13}$ but persistence of improvement at the end of 12 months has confirmed the benefits of intervention. A significant disadvantage of the study is that there is no control group that received no intervention of any sort, but we were obligated to fit in with the local NHS service cycles and to accept a perception of lack of equity in having a control group with no intervention at a time when we had already demonstrated a variety of benefits of the proposed intervention. 13,14,23-25 Among other factors that can influence impact of this booklet are literacy and language. We were not aware of the language and literacy status of our studied population and the booklet was not translated into multiple other languages but, considering that Wolverhampton is an urban area ranked 21st for deprivation in England, ${ }^{26}$ with a 30\% ethnic minority build, is ranked 16th in the UK for poor attainment of qualifications, ${ }^{27}$ moreover the data were tested for the impact 


\section{Key messages}

- Completion of structured diabetes care processes can be influenced by patient-focused interventions

- Provision of patient-specific written information can drive care and improve access to healthcare and diabetes-specific surrogate markers such as $\mathrm{HbA}_{1 c}$

- Improved engagement and better access can be taken as indirect measures of patient activation, which in itself should be an outcome in diabetes care

of deprivation score which did not have an impact on our findings and probably adds to the strength of our findings. The strength of the study also includes its minimal loss to follow-up and the cluster randomisation methodology used to provide robust evidence in this evidence-deficient arena of diabetes care.

In summary, in a large randomised controlled trial, we have demonstrated that the provision of structured diabetes-specific clinical information, through a specifically designed booklet, led to significant improvements in diabetes process outcomes. We also show impact on the long-term measure of glucose control. We are not aware of any previously published randomised controlled trial in diabetes of a whole health economy intervention that is evidence to lead to patient activation.

\section{Implications for research and practice}

We conclude that people with diabetes are manifestly able to understand their most important diabetes-related information when it is presented to them in a simple but structured format and that this promotes their activation in discernible and measurable outcomes. The booklet is easy to generate and is seemingly low tech, with the proviso that the enabling background complexities of data integration and quality assurance are at a very high and well governed standard. Thus, it should be easily reproducible in other health economies. It can be disseminated independently of healthcare professionals, and so is not reliant on service activation nor dogged by service inertia, and it can be systematically distributed across a whole population.

\section{Conflict of interest None declared}

Funding British Medical Association (BMA) Joan Dawkins Grant 2012 partly funded this project but has no role in the writing of manuscript or decision to submit it for publication.

\section{References}

1. Funnell MM, Anderson RM, Arnold MS, et al. Empowerment: an idea whose time has come in diabetes education. Diabetes Educ 1991;17:3741. https://doi.org/10.1177/014572179101700108

2. Anderson RM, Funnell MM, Butler PM, et al. Patient empowerment: results of a randomized controlled trial. Diabetes Care 1995;18:943-9. https://doi.org/10.2337/diacare.18.7.943

3. Norris SL, Lau J, Smith SJ, Schmid CH, Engelgau MM. Self-management education for adults with type 2 diabetes: a meta-analysis of the effect on glycemic control. Diabetes Care 2002;25:1159-71.

https://doi.org/10.2337/diacare.25.7.1159

4. Hibbard JH, Gilburt H. Supporting people to manage their health. An introduction to patient activation. The King's Fund, 2014 http://www.kingsfund.org.uk/sites/files/kf/field/field_publication_file/sup porting-people-manage-health-patient-activation-may14.pdf (accessed 8 Jan 2015).

5. Tricco AC, Ivers NM, Grimshaw JM, et al. Effectiveness of quality improvement strategies on the management of diabetes: a systematic review and meta-analysis. Lancet 2012;379:2252-61. http://dx.doi.org/10.1016/S0140-6736(12)60480-2

6. Hibbard JH, Mahoney ER, Stock R, Tusler M. Do increases in patient activation result in improved self-management behaviors? Health Serv Res 2007;:42:1443-63. http://dx.doi.org/10.1111/j.1475-6773.2006.00669.x

7. Hibbard JH, Mahoney E, Stockard J, Tusler M. Development and testing of a short form of the Patient Activation Measure (PAM). Health Serv Res 2005;40(6, Pt 1):1918-30. http://dx.doi.org/10.1111/j.1475-6773.2005.00438.x

8. National Diabetes Audit 2012-13 Report 1, Care Processes and Treatment Targets. Available at: http://www.hscic.gov.uk/catalogue/ PUB14970/nati-diab-audi-12-13-care-proc-rep.pdf (accessed 15 Jan 2016).

9. Diabetes UK. State of the Nation (England). Challenges for 2015 and beyond. https://www. diabetes.org.uk/Documents/About\%20Us/ What $\% 20$ we $\% 20$ say/State $\% 20$ of\%20the\%20nation\%202014.pdf (accessed 15 Jan 2016)

10. Brookfield SD. Understanding and facilitating adult learning. Buckingham: Open University Press, 1986.

11. Department of Health. Better Information, Better Choices, Better Health: Putting information at the centre of health. London: Department of Health, 2004.

12. Department of Health. Care Planning in Diabetes. Report from the Joint Department of Health and Diabetes UK Care Planning Working Group, 2006. https://www. diabetes.org.uk/documents/reports/careplanningdec06.pdf (accessed 22 Jan 2016).

13. Gillani SMR, Nevill A, Singh BM. Provision of structured diabetes information encourages activation amongst people with diabetes as measured by diabetes care process attainment: the WICKED Project. Diabet Med 2015;32:865-71. http:dx.doi.org/10.1111/dme.12737

14. Gillani SMR, Nayak AU, Thiruvenkatasamy K, et al. A method to validate the accuracy of a centralised district diabetes register. Practical Diabetes 2013;30:224-8. https://doi.org/10.1002/pdi.1780

15. Nam S, Chesla C, Stotts $N$, et al. Barriers to diabetes management: patient and provider factors. Diabetes Res Clin Pract 2011;93:1-9. http:dx.doi.org/10.1016/j.diabres.2011.02.002

16. Shah BR, Hux JE, Laupacis A, Zinman B, Van Walraven C. Clinical inertia in response to inadequate glycemic control: do specialists differ from primary care physicians? Diabetes Care 2005;28:600-06. https://doi.org/10.2337/diacare.28.3.600

17. Khunti K, Wolden ML, Thorsted BL, Andersen M, Davies MJ. Clinical inertia in people with type 2 diabetes: a retrospective cohort study of more than 80,000 people. Diabetes Care 2013;36:3411-17. http://dx.doi.org/10.2337/dc13-0331

18. Khunti K, Damci T, Meneghini L, Pan CY, Yale JF. Study of Once Daily Levemir (SOLVE ${ }^{T M}$ ): insights into the timing of insulin initiation in people with poorly controlled type 2 diabetes in routine clinical practice. Diabetes Obes Metab 2012;14:654-61. http://dx.doi.org/10.1111//.1463-1326.2012.01602.x

19. National Institute for Health and Clinical Excellence (NICE). Primary Care Quality and Outcomes Framework Indicator Advisory Committee recommendations 2010. https://www.nice.org.uk/Media/Default/standardsand-indicators/qof\%20indicator\%20key\%20documents/NM14\%20Adv isory\%20Committee\%20Recommendations.pdf (accessed 26 Jan 2016)

20. Mclntosh B, Cameron C, Singh S, et al. Second-line therapy in patients with type 2 diabetes inadequately controlled with metformin monotherapy: a systematic review and mixed-treatment comparison meta-analysis. Open Med 2011;5:e35-48.

21. Khunti K, Gray LJ, Skinner T, et al. Effectiveness of a diabetes education and self management programme (DESMOND) for people with newly 
diagnosed type 2 diabetes mellitus: three year follow-up of a cluster randomised controlled trial in primary care. BMJ 2012;344:e2333. https://doi.org/10.1136/bmj.e2333

22. Hopkins D, Lawrence I, Mansell P, et al. Improved biomedical and psychological outcomes 1 year after structured education in flexible insulin therapy for people with type 1 diabetes: the UK DAFNE experience. Diabetes Care 2012;35:1638-42. http://dx.doi.org/10.2337/dc11-1579

23. Gillani SMR, Holland M, Sidhu M, Singh BM. A case control study of use of the Failed Access Score for determination of failed access to structured diabetes care: the WICKED project. Practical Diabetes 2014;31:107-10. http://dx.doi.org/10.1002/pdi.1843

24. Gillani SMR, Singh BM. A simple method for introducing care planning into specialist diabetes clinics. The WICKED project. Practical Diabetes
2014;31:337-41. http://dx.doi.org/10.1002/pdi.1898

25. Gillani SMR, Nevill A, Singh BM. The evaluation by people with diabetes of the usefulness of a mail delivered personalised diabetes information booklet with insight into the association of patient activation to clinical risk: the WICKED Project. http://dx.doi.org/10.15277/bjd.2016.099

26. Department for Communities and Local Government. The English Indices of Deprivation 2015. https://www.gov.uk/government/uploads/system/ uploads/attachment_data/file/465791/English_Indices_of_Deprivation_2 015_-_Statistical_Release.pdf (accessed 5 June 2017).

27. Uneducated Britain - how does your constituency compare? https://www.theguardian.com/news/datablog/2011/jul/22/educationgap-by-constituency (accessed 5 June 2017).
We've come

together to deliver:

THE WORLD'S PREMIER DIABETES MANAGEMENT PLATFORM glooko + diasend.

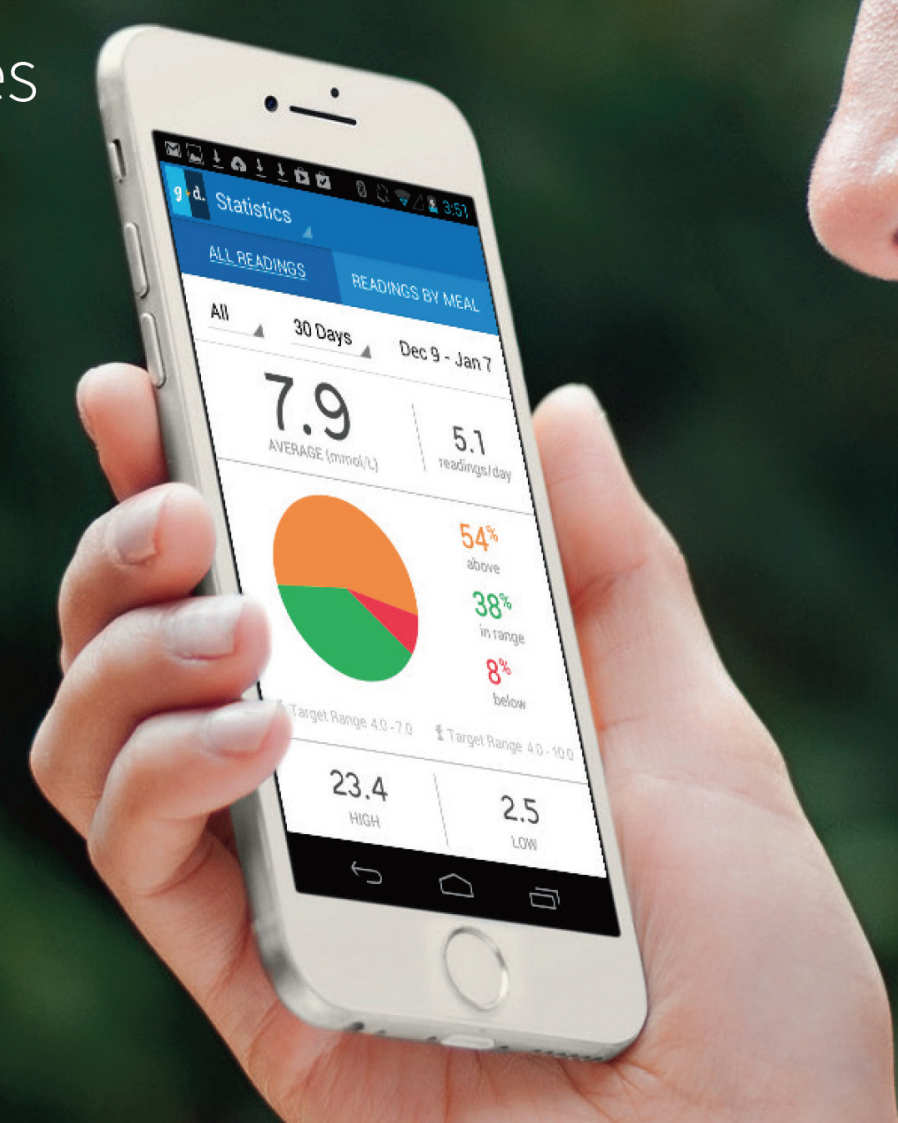

